\title{
MOBILE HEALTH AWARENESS IN PRE-DETECTION OF MILD STROKE SYMPTOMS
}

\author{
Oi-Mean Foong, Jing-Mei Yong, \\ Suziah Sulaiman and Dayang Rohaya Awang Rambli \\ Department of Computer and Information Sciences, \\ Faculty of Science and Information Technology, Universiti Teknologi PETRONAS, Malaysia
}

Received 2014-04-29; Revised 2014-07-30; Accepted 2014-11-24

\begin{abstract}
Stroke contributes to the third leading cause of death in Malaysia and happens to be the prime cause of disability among Malaysian. Lack of awareness and knowledge of stroke among Malaysian have often led to delay in hospital administration and reduced the chances of recovery for stroke patients. The objectives of this research are three-fold: (1) To develop a mobile application that helps to perform early detection on mild stroke symptoms (2) To increase the stroke awareness and knowledge level among the society (3) To create a reminder to constantly motivate the society to perform regular selfcheck on mild stroke symptoms. The mobile application is targeted for general public usage. Interviews were conducted with consultant neurologist and physiotherapist \& rehabilitation manager of the National Stroke Association of Malaysia (NASAM). An online pre-survey has also been conducted among 70 random people to investigate the feasibility of the mobile application. The stroke predetection application was implemented in Android platform. The National Institute of Health Stroke Scale (NIHSS) will be used as a guideline for the development of self-check assessment for detecting mild stroke symptoms as it is proven to be reliable and effective evaluation technique. Empirical results show that majority of the respondents believe that the mobile stroke application can increase their stroke awareness and help them to perform early detections on mild stroke symptoms. The mobile stroke application performs satisfactorily in terms of its usefulness, usability, mobile application quality and information quality.
\end{abstract}

Keywords: Mild Stroke Symptoms, National Institute of Health Stroke Scale, Self-Check Assessment

\section{INTRODUCTION}

Approximately 15 million people across the globe experience stroke annually whereas 5 million of them have ceased and another 5 million of them were paralyzed infinitely (Stroke Centre, 2013). According to the National Stroke Association Malaysia, 1 in 6 people worldwide will encounter a stroke along their life span. In Malaysia, stroke contributes to the third leading cause of death and severe disability among the society (NSAM, 2013).
Stroke is commonly addressed as sudden brain attack that takes place when the blood supply to the brain is disrupted which leads to the deficiency of oxygen and glucose supply to the brain cells. Stroke can be categorized into ischemic stroke and haemorrhagic stroke. About $80 \%$ of the strokes are ischemic stroke, where the blood vessel in the brain suffers a clot that terminates the blood flow to brain cells. The remaining $20 \%$ are haemorrhagic stroke, which is due to the damage and outbreak of weakened

Corresponding Author: Oi-Mean Foong, Computer and Information Sciences Department, Universiti Teknologi PETRONAS, Malaysia 
blood vessel in the brain (WebMD, 2012). Time is an essence in stroke attacks. An individual that has been identified with stroke symptoms should be administered to hospital immediately for treatment to prevent subsequent deterioration of individual's life (Holtmann et al., 2007). The main problem associated with underutilization of stroke treatment is mainly due to late hospital administration of potential stroke patients. Previous researches demonstrated that 15 $60 \%$ of patients seek hospital treatment around $3 \mathrm{hr}$ after the detection of stroke symptoms and only 14$48 \%$ of patients arrive to hospital within $2 \mathrm{hr}$ (Fassbender et al., 2013). The common cause of delay in hospital administration among the society is due to limited knowledge level and awareness of the severity of stroke. Various studies have identified that the delay in hospital administration is largely related to the inability to determine the stroke symptoms and the adoption of a wait-and-see attitude. In addition to various educational campaigns held to raise the awareness of stroke, the impact that has been derived from these activities is minimal if there is no established platform to motivate and remind people about stroke risks (Fassbender et al., 2013).

This research was motivated by the fact that there exists no established mobile application of prestroke management in Malaysia based on literature study. It is believed that there is a massive potential to implement telemedicine in the field of stroke especially for pre-detection of mild stroke symptoms. The objectives in this paper are three-fold: (1) To develop a mobile application that helps the society to perform early detection of mild stroke symptoms. (2) To increase the stroke knowledge and awareness among Malaysian society. (3) To constantly remind and motivate individual to perform regular self-check on mild stroke symptoms using an alert reminder.

Mild stroke, which is commonly known by many as "mini stroke", has the scientific name of Transient Ischemic Attack (TIA). The mild stroke may be categorized by some loose terms such as isolated sensory loss, ataxia, facial weakness, or dysarthria (Urra et al., 2013). It is actually similar to stroke, however, it only lasts for a short period of time, usually less than 24 hours (Fischer et al., 2010). Unlike a stroke, the symptoms of mild stroke (TIA) will disappear quickly. Similarly, (Fischer et al.,
2010; Khatri et al., 2010) discovered that mild stroke is non-disabling. However, it is of utmost importance to seek for medical attention once mild stroke symptoms is discovered, as it will lead to an increased risk in experiencing a severe stroke in the near future.

The National Institute of Health Stroke Scale (NIHSS) is widely adopted in the western countries as a measurement of mild stroke symptoms (Kasner, 2006). (Urra et al., 2013; Khatri et al., 2010) stated that mild/minor stroke is commonly defined as clinical deficits measured on the NIHSS with score $\leq 5$ in most research studies. (Schwamm et al., 2009) demonstrated that NIHSS is commonly applied as a standard guideline for stroke clinical deficit scale assessment as the 13-item graded neurological examination covers almost all the assessment for stroke signs. According to (Zandieh et al., 2013; Berthier et al., 2013), the examination of stroke patients were often conducted based on the NIHSS scale as it is reliable in terms of its competency in anticipating the patient's outcome. Hence, it is recommended to be used in various clinical settings.

In view of stroke symptoms among the high risk or potential stroke patients, the American Stroke Association has come up with the FAST technique that demands of early detections of stroke. The technique motivates every individual to think and act FAST in identifying stroke symptoms so that it reduces the chances of possible fatality and severe impairment (ASA, 2013a).

The FAST technique used is as follows:

- F-Face an indicator to measure the facial expression. A crooked smile or a drooped mouth would be the signs of having a stroke

- A-Arms an indicator to measure the flexibility of movements in potential stroke patients. If the individual fails to raise one or both arm, it indicates that the person is perhaps having stroke symptoms

- S-Speech an indicator to detect the ability of an individual to speak fluently, repeat some simple sentences or pronounce certain words. Slur speech or inaccuracy in the pronunciations of certain words will be the warning signs of stroke

- T-Time a reminder to urge the society to contact the emergency personnel or hospital immediately when stroke symptoms are detected 
Other stroke warning signs that appeared in a particular individual are confusion (trouble understanding other people), vision problems (trouble seeing with one/both eyes), trouble staying balance or coordination and dizziness or severe headaches for unknown reasons.

The remaining of the paper is organized as follows. Section 2 is the literature review. Section 3 describes the proposed Mild Stroke Pre-Detection (Stroke PD) System Architecture. A survey was conducted in Section 4 prior to the experimental setup. Section 5 discusses the experimental findings. Section 6 states the conclusion and future work.

\section{LITERATURE REVIEW}

The common methods of creating stroke awareness are through organizing various stroke educational campaigns, word-of-mouth strategy, pamphlets and also through information sharing in social media platforms. In spite of various campaigns of stroke, the response to stroke symptoms management does not translate into appropriate actions for potential stroke patients (Grady et al., 2014; Fassbender et al., 2013). There is an urgent need to leverage telehealth care for the delivery of medical information and services at anytime and anywhere especially during emergency (Schwamm et al., 2009). Based on global market statistics, approximately $78.4 \%$ of smart phone users are running their mobile applications in Android platform (SMSF, 2009-2013). Therefore, we designed and developed Stroke PD application in the Android platform for early detections of mild stroke symptoms.

Some of the existing mobile health awareness applications designed to raise stroke knowledge and awareness include Think F.A.S.T. (http://strokefoundation.com.au, 2013), Spot a Stroke FAST (ASA, 2013b), Brain Attack App (Rajeev, 2013) and Stroke 119 (Nam et al., 2014) and the National Institute of Health Stroke Scale (NIHSS, 2013).

'Think F.A.S.T.' is a simple mobile application National Institute of Health Stroke Scale that is targeted for the general use by public. It could recognize the warning symptoms and locate Australian hospitals with specialized stroke units in times of emergencies. The functions and features of Think F.A.S.T. include providing tips on stroke, recognizing the signs of stroke in plain text basis, quiz to improve the understanding on stroke symptoms and it could locate the nearest Stroke Emergency Care Centre.
'Spot a Stroke FAST' is a mobile application that is targeted for the general use by public. It aims to educate the society on stroke symptoms using a way that is easy to remember. It consists of tips and resources on stroke, recognizes the signs of stroke in plain text basis and video clips on FAST detection of stroke symptoms.

'Brain Attack App' is a mobile application designed for the professionals such as doctors and nurses to conduct systematic and quantitative measurement of neurological deficit. It includes 30 questionnaires for recognizing signs of stroke and to determine the eligibility for Thrombolytic therapy with intravenous tissue Plasminogen Activator (t-PA) treatment. The tissue plasminogen activator can be used to treat some patients who are having a stroke caused by a blood clot (ischemic stroke). In addition, the summary of the results could be printed for further analysis.

'Stroke 119' was developed to aid rapid stroke screening in multi-platforms e.g., iPhone and Android platforms. Unlike the other mobile applications, it consists of cartoon figures for facial palsy, arm weakness and slurred speech. The Stroke 119 app also encompasses a map from the web application so that all hospital map data in Korea are up-to-date.

'The National Institute of Health Stroke Scale' NIHSS is another mobile application for bystanders or emergency medical personnel to help patients in examining their neurological deficits. The application includes a checklist to examine the stroke condition of the patient, results and track the history of user's examination.

Based on literature study, there are still limitations in the existing mobile healthcare applications for stroke. For examples, there is limited existence of effective methods to assess the actual health condition of the potential stroke patients; there is no alarm reminder to constantly motivate the potential stroke patient for regular self-check on stroke symptoms; there is a lack of integration functionality and/or interactions with users in the existing mobile applications and there is also no tracking history for the results of regular selfcheck assessment on stroke symptoms. Therefore, Stroke PD is an essential tool in raising the stroke awareness and knowledge among the society. The functionalities that would be implemented in the Stroke PD mobile application includes (1) a comprehensive self-check assessment that is based on NIHSS stroke scale which allows user to perform early detections of mild stroke symptoms independently and in a costeffective way. (2) An alarm reminder to constantly 
remind and motivate users to perform regular self-check assessment on mild stroke symptoms. The integration of various functionalities and features in Stroke PD mobile application would increase the interactions with users and also the user-friendliness aspect for the mobile application. (3) Summary results that keep track of history of regular self-check assessment.

\section{PROPOSED SYSTEM ARCHITECTURE}

The proposed system architecture of Stroke PD mobile application is shown in Fig. 1. It consists of 4 main processes: Stroke Info, Self-check Assessment, Alarm Reminder and Emergency Contact Button. The core functionality of Stroke PD mobile application would be the self-check assessment and the alarm/warning alert/reminder to constantly motivate and remind the users to perform regular self-check for mild stroke symptoms. The self-check assessment comprises three sections, namely consciousness test, mobility test and self-check questions. Users will have to perform all the three sections for the self-check assessment. The answers will be further processed and accumulated based on the National Institute of Health Stroke Scale (NIHSS). The results of the self-check assessment will then be stored into the database for future tracking purposes. In the meantime, result of the self-check assessment will also be displayed to the user in order to alert them on their health condition.

The Stroke PD mobile application is targeted for the use of general public, especially for the potential stroke patients. The functions and features comprise of the following items:

- Resources on stroke information

- Real life testimonials of stroke patients

- Video clips on stroke messages delivered by physiotherapist of NASAM and to guide users on the actions needed to perform regular for self-check of mild stroke symptoms

- Two interactive self-check features on consciousness and mobility impairment test

- Audio recording to narrate questions for the selfcheck questionnaires to enhance usability

- Nine self-check questionnaire based on NIHSS scoring method

- Tracking history for the results of self-check assessment

- Alarm reminder
- Emergency call 999 and contact family member immediately

The stroke system prototype was designed and developed in Android Jellybean 4.2 within the Eclipse IDE. It was further tested on Android emulator and deployed on Samsung S4 smartphone.

\section{RESULTS}

A pilot study was performed before the development of Stroke PD mobile application with the aim to obtain comprehensive information related to stroke as well as the desired functionalities for the targeted users.

\subsection{Pre-Survey Interview Sessions}

An interview session had been carried out with the consultant neurologist of ANOC Neuroscience and Orthopaedic Centre, which is prominently located in Bangsar, Kuala Lumpur. According to the neurologist there is no stroke scales adopted by hospitals in Malaysia for stroke evaluation. The condition of stroke patients is mainly determined by their medical histories. Unlike the advanced countries, pre-hospital stroke management process does not exist in Malaysia. Malaysian often experience delay in seeking medical treatment especially for stroke diseases due to limited state-of-the-art technology to assist in creation of stroke awareness and stroke symptoms detection in potential patients.

In addition, another interview session had also been conducted at the National Stroke Association of Malaysia (NASAM). In contrast to earlier interview, NASAM focuses only on the post-stroke management process and does not involve in any pre-stroke management. However, various stroke education campaigns such as awareness talks and stroke awareness day were held to raise the awareness and knowledge of stroke in Malaysia. Based on literature study, there is no existing technology to assist healthcare officer in delivering stroke messages to general public. Stroke messages are usually conveyed to the general public through verbal communications via road shows. Hence, this has motivated us to leverage on healthcare mobile application to facilitate NASAM to disseminating stroke awareness and/or messages to the public.

\subsection{The Pre-Survey Results}

An online pre-survey session was conducted with 70 respondents using 5-point Likert scale where 1 indicates least favourable and 5 indicates the most favourable. The 
aim of the study is to investigate the knowledge level of stroke of general public, the awareness of stroke among the society and the factors that contribute to the delay in hospital administration.

Fig. 2 shows the responses of user's experiencing delay in hospital administration. Out of the 13 respondents who have a family history for stroke, it was found that 10 of them have experienced delay in sending their family members to hospital for stroke. This has demonstrates that there is low awareness and limited stroke knowledge among the society as they are unable to detect the stroke symptoms in their family members in a timely manner. Therefore, it is crucial to detect stroke symptoms very quickly before the stroke patient becomes permanently disabled or becomes fatal.

Fig.3 demonstrates the response of users upon detection of stroke symptoms. It was observed that 10 out of the 13 respondents who have detected stroke symptoms in their family members adopt a wait-andsee attitude rather than seeking for immediate medical attention. This might be due to the insufficiency of stroke knowledge and subsequently cause them to be unsure of what to do upon detection of stroke symptoms. Hence, it has inspired us to embark on this healthcare research on development of Stroke PD mobile application to enhance the stroke knowledge level and awareness among Malaysian society.

\subsection{Stroke PD User Interface Design}

The Stroke Pre-detection (Stroke PD) mobile application consists of various components which allow users to interact with and choose from main menu. Fig. 4 illustrates the user interface for the start-up screen and home screen for Stroke PD mobile application. The home screen consists of 4 main functions: Stroke Info, Self-check Assessment, Alarm Settings and Emergency Contact.

All stroke information and stroke risk factors are displayed in Fig. 5(a). It consists of stroke articles on "What is stroke" and "Stroke risk factor" whereas "Stroke symptoms" identification is done using FAST technique (ASA, 2013a) as illustrated in Fig. 5(b).

How to prevent stroke? The best way to prevent a stroke is to reduce the risk factors associated with strokes. The controllable stroke risk such as hypertension was found out to be the major contributing factor for stroke in Malaysia. Other stroke risk factors are namely diabetes mellitus, Transient Ischemic Attack (TIA) or mild stroke active smoking, excessive, physical and obesity (Nazifah et al., 2012).
In this section, the warning signs of stroke using FAST technique were illustrated in Fig. 5(b). They are (i) having a crooked smile on one's Face, (ii) weakness on the Arms, (iii) slur Speech and (iv) Time to call 999 for stroke check-up.

It is strongly belief that real life testimonies would create a strong awareness to the community on the impacts of neglecting stroke symptoms. It is hoped that the real life testimonials will raise the awareness of stroke. In addition, a video clip on stroke is embedded into the Stroke PD application for physiotherapist to share her stroke knowledge.

The self-check assessment as shown in Fig. 6(a) and Fig. 6(b) act as the core functionality of Stroke PD mobile application by helping user to perform early detections of mild stroke symptoms. It consists of 3 main sections namely consciousness test in section A; mobility test in section B; facial palsy, speech, gaze, vision, arms, legs, dizziness and balancing tests in section $\mathrm{C}$. Consciousness test is an interactive game that measures the consciousness level in an individual. Three different pictures will be randomly generated and users will have to choose the correct answer based on the given question. For every correct answer, zero score will be allotted and it indicates that the user is in his conscious state. Otherwise, score of 1 will be allotted for every wrong answer.

The second part of the self-check assessment is on mobility testing. Mobility test helps to determine the flexibility issue in an individual through an interactive game for users to key in the 4-digit number. If the time taken to enter the digits exceeds a certain time limit, then it indicates that there might be flexibility issue with the user, which also represents one of the signs of mild stroke symptoms. Besides, the accuracy of the answer will also be taken into account. Upon completion of the mobility test, there will be a series of self-check questionnaires related to mild stroke symptoms which require users to perform independently. For instance, users will be asked to perform self-check on facial palsy. The user will need to check and determine whether they can smile properly or having a crooked smile. The users will have to answer accordingly based on their ability to perform the assigned activities.

After the completion of self-check assessment, the scores will be accumulated and compiled together based on the pre-defined scores based on the NIHSS stroke scale. If the accumulated score is 1 , the user will be regarded as having mild stroke symptoms. However, if the user's score is 0 , it will display the zero score and congratulates him for being pink in the health. 


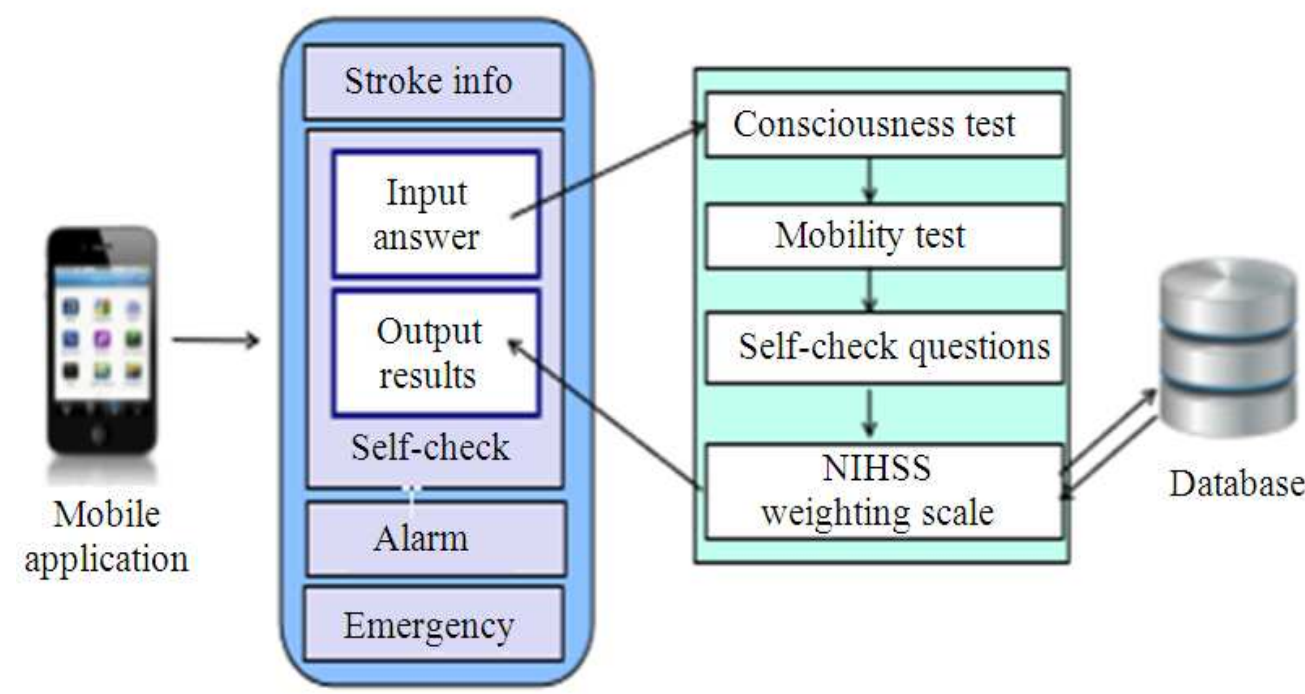

User interface

Fig. 1 System architecture of stroke PD mobile application

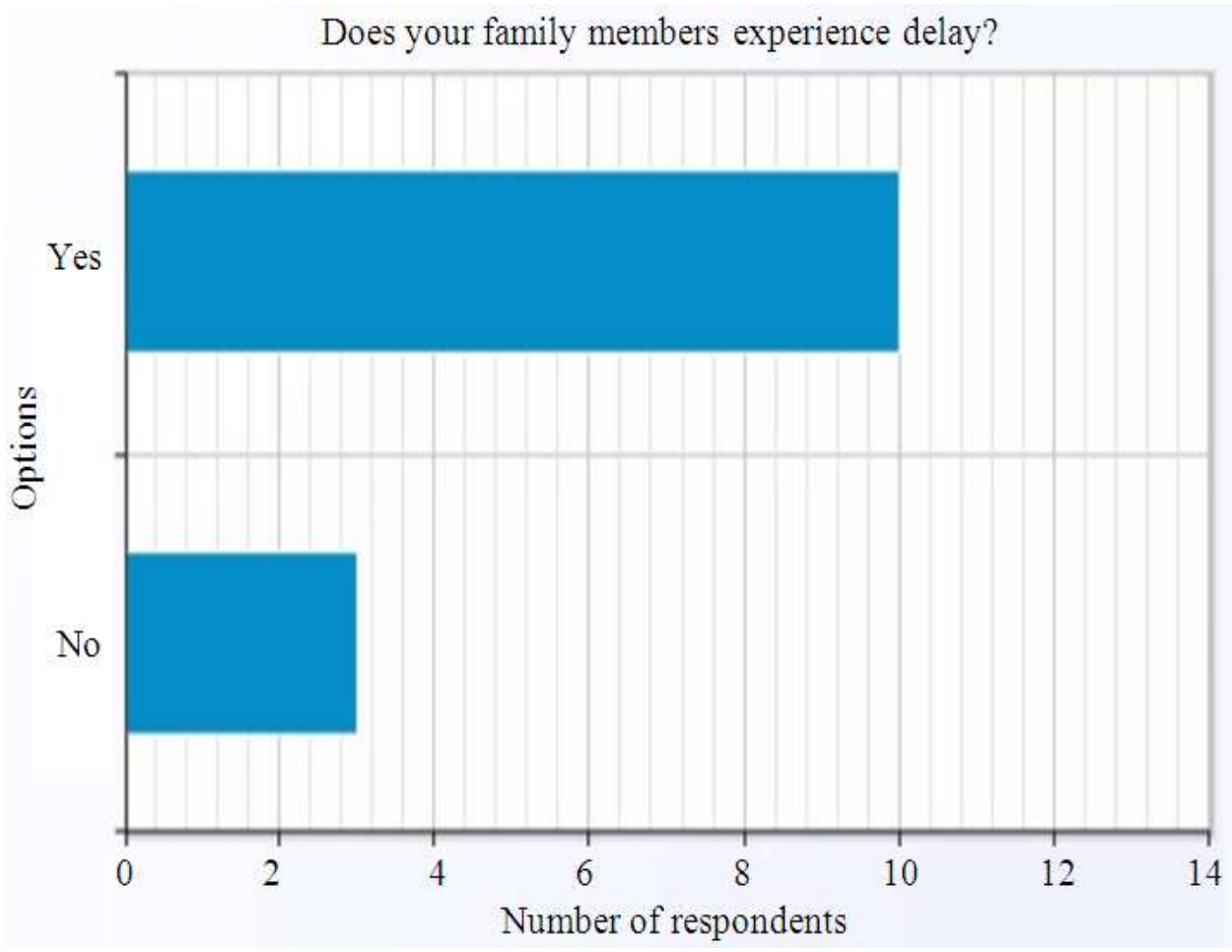

Fig. 2 User's experience in delay for hospital administration 
Oi-Mean Foong et al. / Journal of Computer Science 10 (12): 2383.2394, 2014

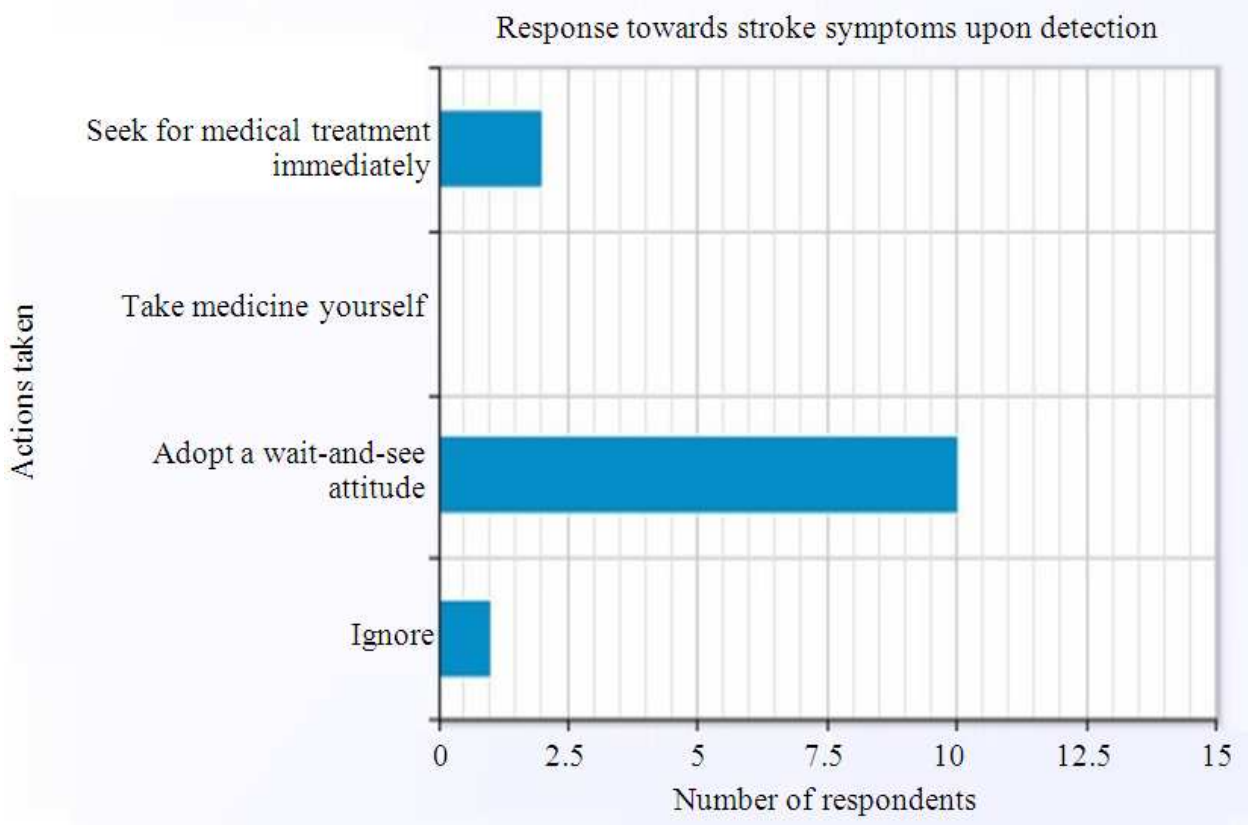

Fig. 3 Response of users upon detection of stroke symptoms

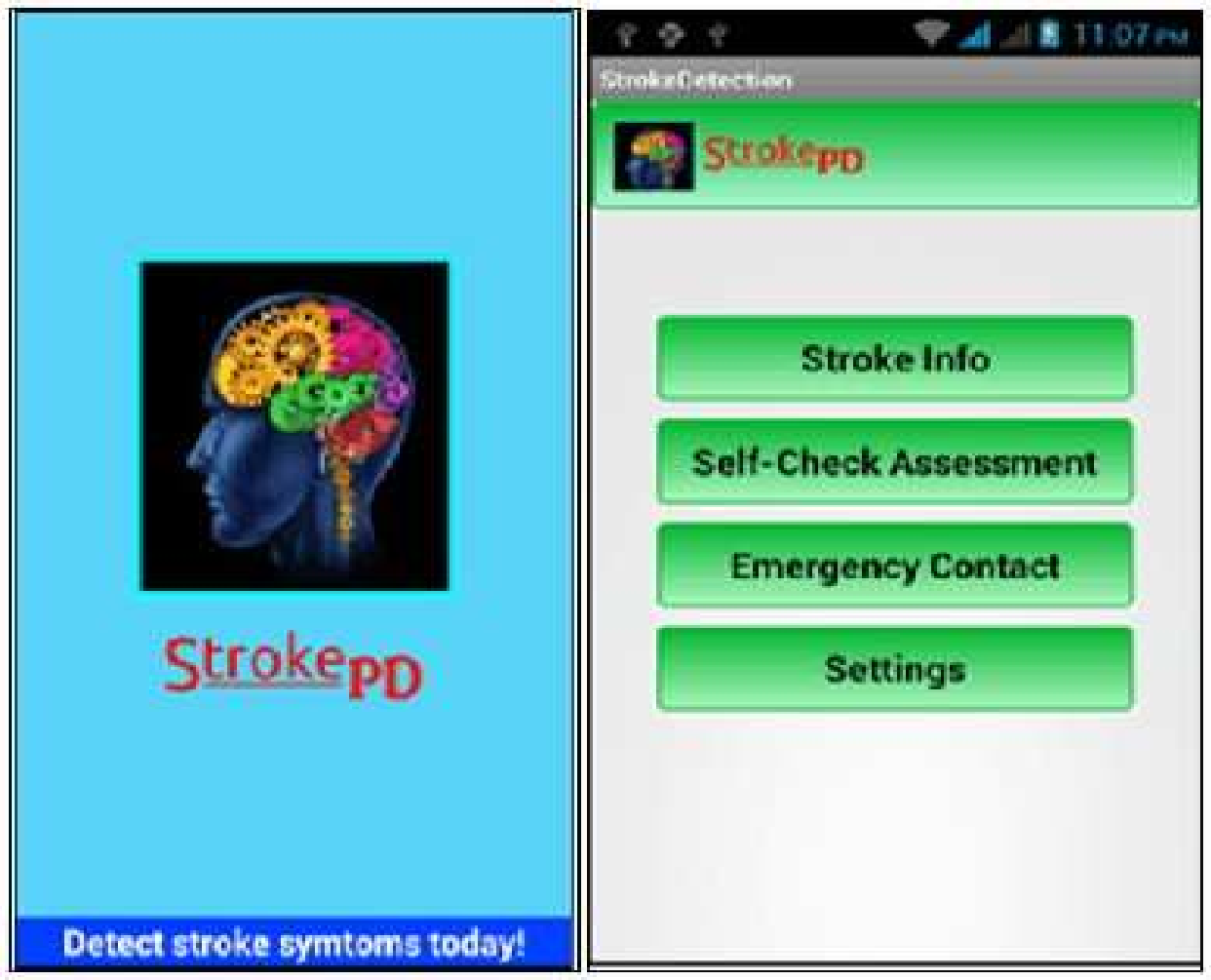

Fig. 4 Stroke PD main menu 
Oi-Mean Foong et al. / Journal of Computer Science 10 (12): 2383.2394, 2014

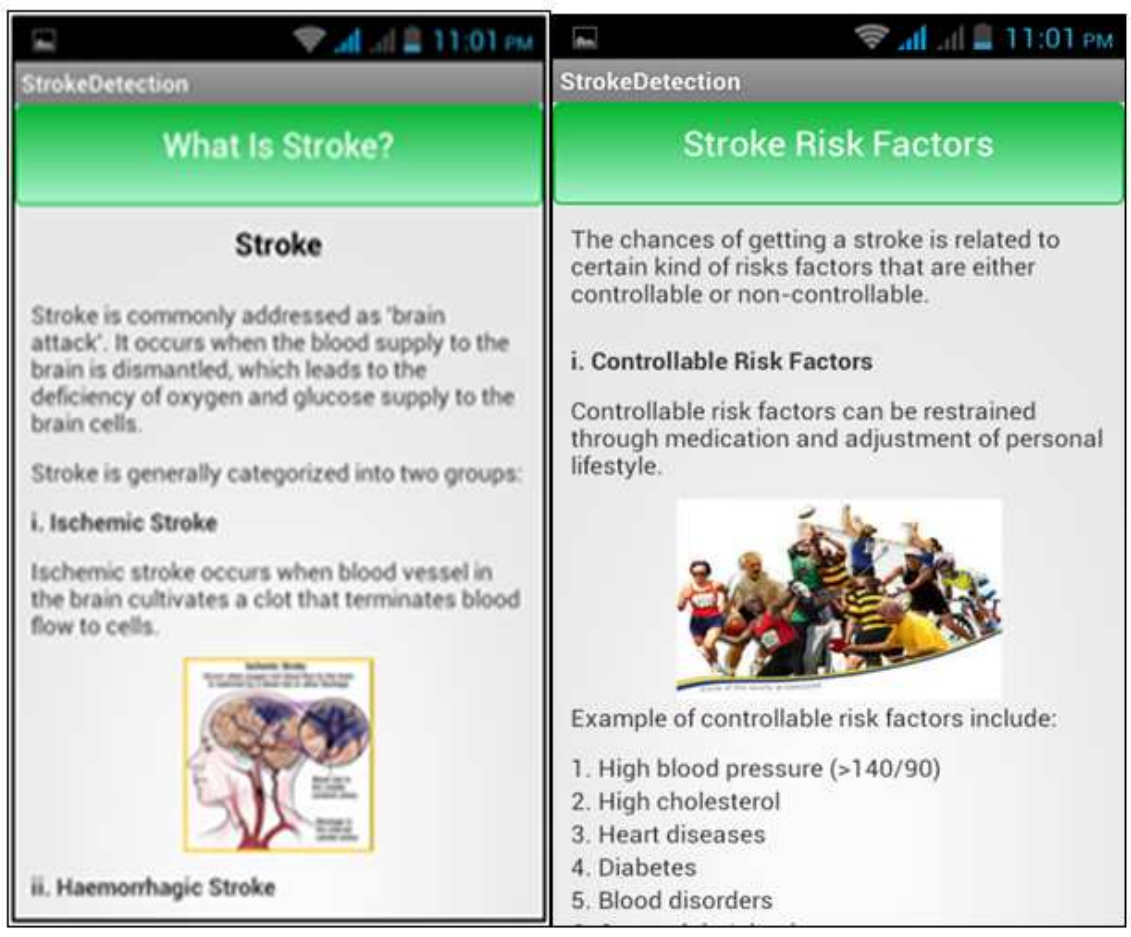

Fig. 5(a) Stroke and risk factors (http://www.patient.co.uk/)

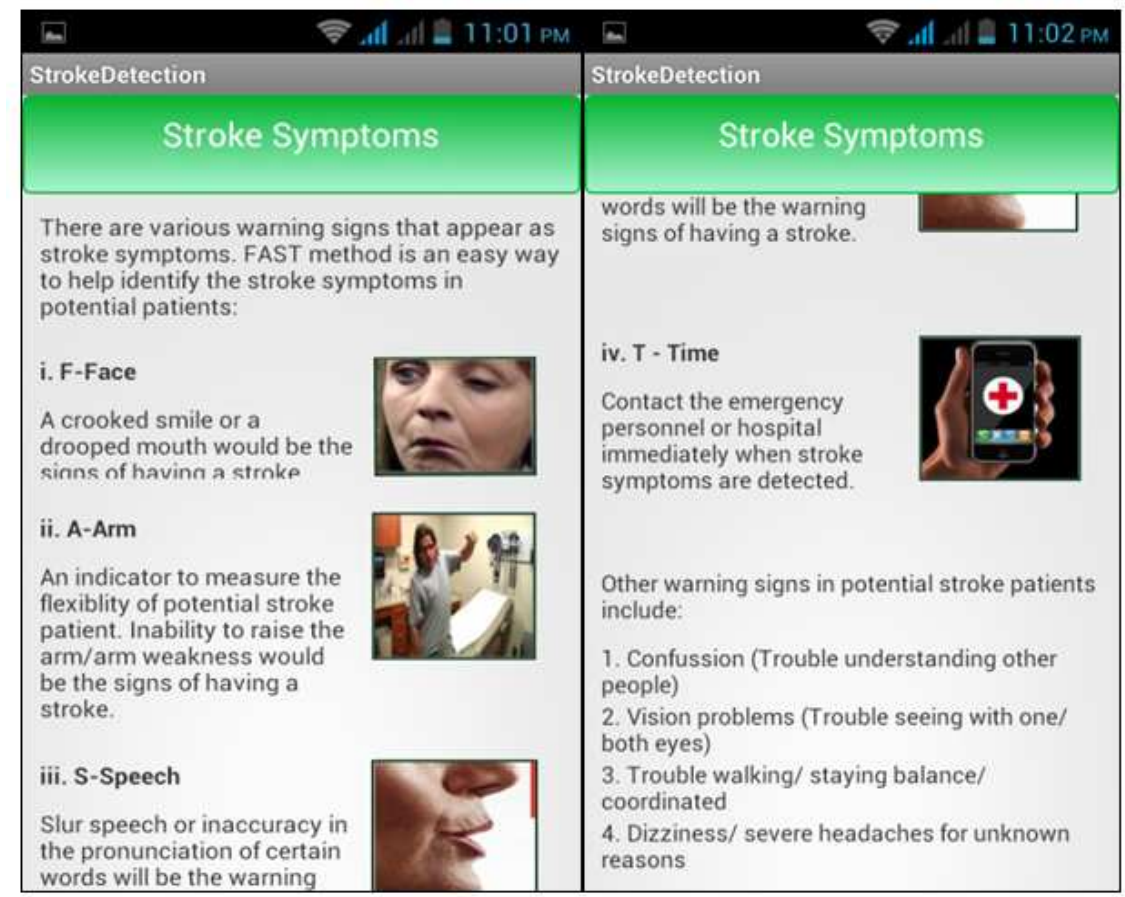

Fig. 5 (b) FAST technique to identify stroke symptoms (http://www.patient.co.uk/) 


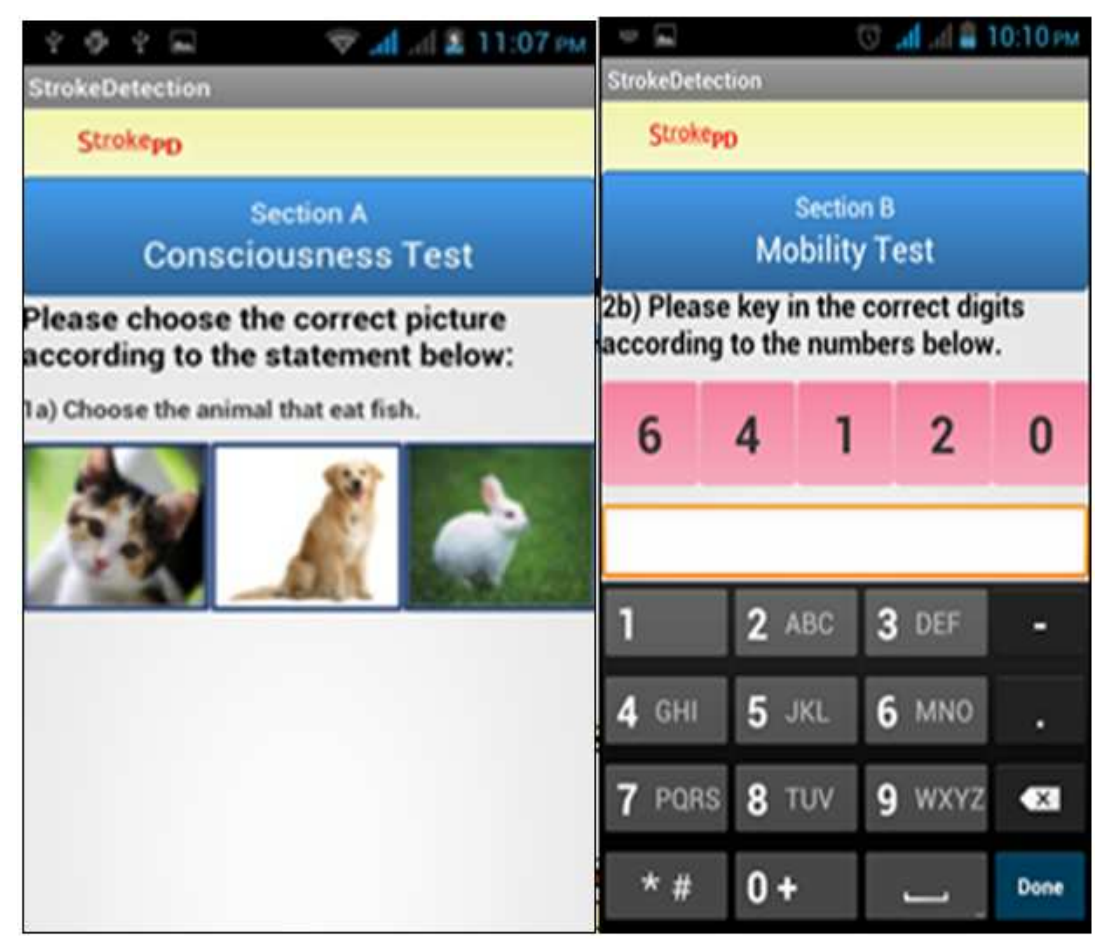

Fig. 6 (a) Consciousness and mobility tests

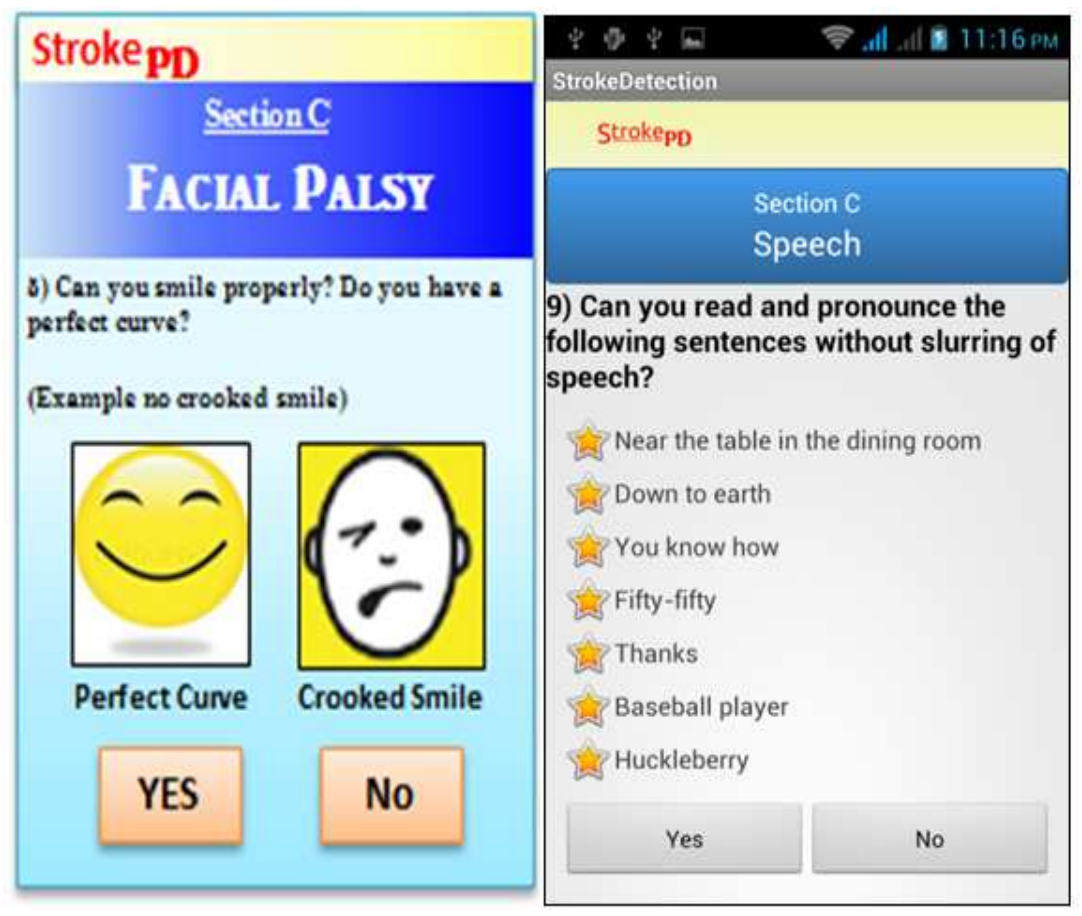

Fig. 6 (b) Facial palsy and speech tests (Think, 2013) 


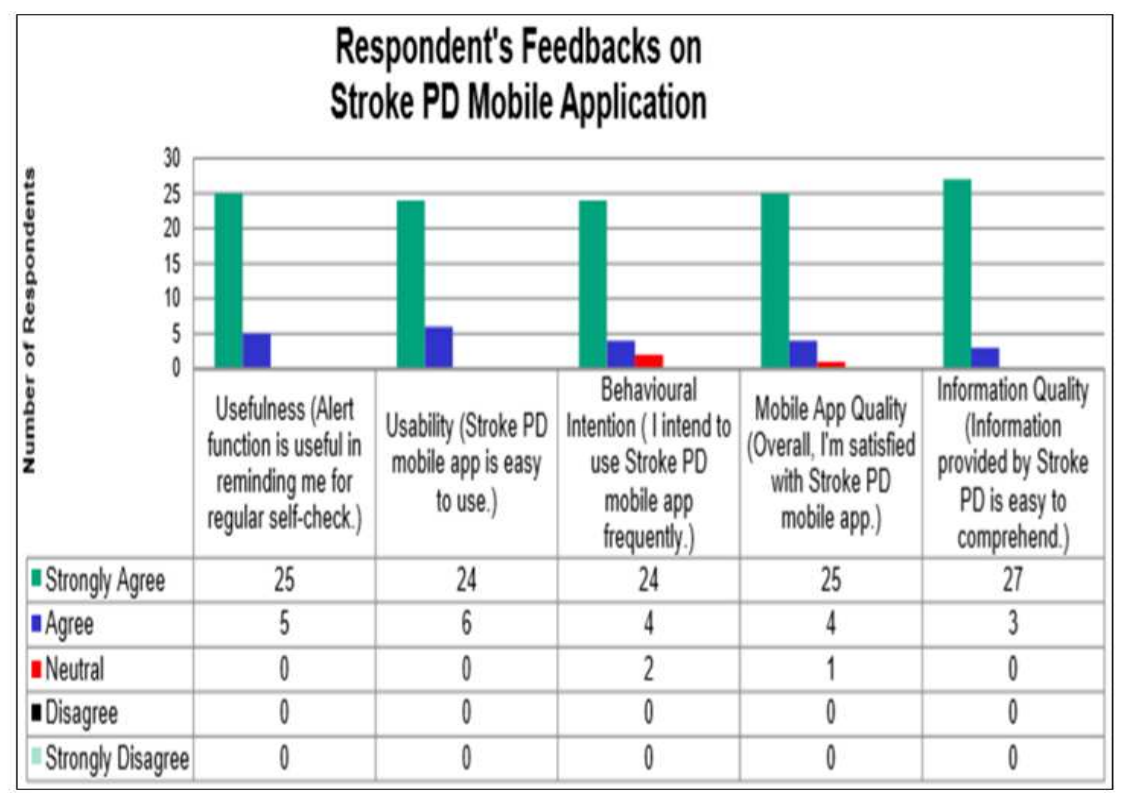

Fig. 7 Stroke PD system evaluation results

In contrast, if users are having $1 \leq$ score $\leq 5$, then they are likely to have mild stroke symptoms whereas a score that exceeds 5 indicates that the user might have a severe stroke. The users are advised to visit the hospital immediately for subsequent check-ups regardless of their total scores if they are suspected of having stroke. The compiled scores and the related details will also be stored in the self-check history database along with the time stamp for future references. Hence, users can track their medical history from time to time for health monitoring purposes.

In case he/she is suspected of having stroke, he could immediately call the emergency number 999 for immediate assistance. Alternatively, he could contact his family member for help with just a click of the button. Last but not least, there will also be alarm settings to alert users to set their alarm to constantly remind and motivate them to perform regular self-check on mild stroke symptoms.

\subsection{Stroke PD System Performance Testing}

The system performance testing has been carried out upon completion of Stroke PD prototype and the main objectives are:

- To perform testing for each specification in Stroke

- To measure user's perception and acceptance level
The respondents for the system performance testing were targeted to two different groups as listed below:

- Experts: The physiotherapist of National Stroke Association of Malaysia was invited for the end-toend functionality testing and to provide feedbacks on Stroke PD in terms of its usefulness, usability, information quality as well as its ability to increase stroke awareness among the Malaysian society

- Users: 30 random respondents from different backgrounds were selected to operate Stroke PD mobile application and subsequently, provide their users' learning experience through answering a set of designed questionnaires. The respondents were chosen randomly and post-test questionnaires were distributed

- Ten young adults from the age range of 13-30

- Ten middle-aged persons from the age range of 30-50

- 10 old-aged people with the age of 50 and above

Prior to the evaluation of Stroke PD mobile application, a short briefing was given to all the participants to ensure they understand the aim of the system testing and the respective functionalities in Stroke PD. The respondents will subsequently rate their users' experiences in the designed questionnaires and provide their perceptions of the usability testing based on 5-point Likert scale. 


\section{DISCUSSION}

The system performance evaluation was conducted and its evaluation results were illustrated in Fig. 7. It was observed that majority of the respondents agree that Stroke PD mobile application was very useful to them in terms of raising their stroke knowledge and awareness. The alert function in the proposed system was found useful by the respondents in terms of reminding and motivating them to perform self-check assessment on mild stroke symptoms frequently.

The majority of the respondents claimed that Stroke PD mobile application is easy to navigate and user friendly. Among the 30 respondents interviewed, 93.3\% of the respondents recognize that it is easy to perform pre-detection of mild stroke symptoms using Stroke PD mobile application as it contains video to guide them step-by-step for their regular self-check.

The majority of the respondents also stated that it was easy for them to navigate through the screens of Stroke PD mobile application. In general, most of the respondents perceived that Stroke PD mobile application was easy to use to achieve their goals. The tabulated results have significantly reflected the positive behaviour of the respondents in using Stroke PD mobile application as illustrated in Fig. 7. Most of the respondents intend to use Stroke PD mobile application as often as they need especially in terms of helping them to perform early detection on mild stroke symptoms. Furthermore, most of them positively state that they will strongly recommend their family members, relatives and friends to use Stroke PD mobile application in order to raise their stroke knowledge level and awareness in them. A wide percentage of the respondents, which total up to $96.7 \%$ (confidence level \pm 0.05 ) agreed that the overall quality for Stroke PD mobile application was excellent and they were satisfied with the proposed system.

Despite the fact that the Stroke PD mobile application has brought about multitude of benefits to general public in Malaysia, it has some drawbacks in the prototype system. The current mobile application fails to alert the emergency contacts of nearby hospitals and National Stroke Association Malaysia if the potential stroke patients reside in remote areas that are without mobile phone network coverage.

\section{CONCLUSION}

The Stroke PD mobile application is effective to raise stroke awareness and stroke knowledge level among the
Malaysian society. The self-check assessment and alarm reminder constantly remind and motivate users to perform early detections of mild stroke symptoms. It will undoubtedly prevent and reduce the risks of disabilities or impairment and even fatal death whenever onset of stroke occurs. The system performance testing demonstrated that the Stroke PD mobile application is highly favoured in terms of its usefulness, usability, mobile application quality and information content. It is a valuable tool that helps to perform early detection of mild stroke symptoms, increase the stroke knowledge and awareness among the Malaysian society and serves as a constant reminder to give warning/alert for any individuals to perform regular selfcheck of mild stroke.

For future work, user could take picture from his smart phone camera and apply image recognition technique for detection and recognition of facial palsy for crooked smile. This will eventually increase the effectiveness in the detection of facial palsy (crooked smile) among potential stroke patients as there will be no bias in the facial recognition. Furthermore, the Stroke PD application could be re-designed in other languages for better accessibility.

\section{ACKNOWLEDGEMENT}

We would like to thank the National Stroke Association of Malaysia (NASAM) and Universiti Teknologi PETRONAS for the support in this STIRF research.

\section{ADDITIONAL INFORMATION}

\subsection{Funding Information}

This project was funded by Research Innovation Office at Universiti Teknologi PETRONAS.

\subsection{Author's Contributions}

Oi-Mean Foong: She was responsible for the conception, design, analysis of the results, deployment and revamp of the manuscript .

Jing-Mei Yong: She was in charge of the coding, testing and development of the Android application.

Suziah Sulaiman: She reviewed, analyzed and revised the manuscript.

Dayang Rohaya Awang Rambli: She reviewed, analyzed and revised the manuscript.

\subsection{Ethics}

All informations about stroke are disseminated, cited 
and adhere with the norm and professional conduct of scholarly writing for publication. However, the information does not constitute advice and all users are advised to seek professional medical assistance in the event that they are suffering from any medical problems such as stroke.

\section{REFERENCES}

ASA, 2013a. Warning signs of stroke. American Stroke Association.

ASA, 2013b. Spot a stroke F.A.S.T. American Stroke Association.

Berthier, E., P. Decavel, F. Vuillier, C. Verlut and E.D.B. Medeiros et al., 2013. Reliability of NIHSS by telemedicine in non-neurologists. Int. J. Stroke, 8: 11-11. DOI: 10.1111/j.1747-4949.2012.00965.x

Fassbender, K., C. Balucani, S. Walter, S.R. Levine and J. Grotta et al., 2013. Streamlining of prehospital stroke management: The golden hour. Lancet Neurology, 12: 585-596. DOI: 10.1016/S01406736(08)61345-8

Fischer, U., A. Baumgartner, M. Arnold, K. Nedeltchev and H.P. Mattle et al., 2010. What is a minor stroke. Stroke, 41: 661-666. DOI: 10.1161/ STROKEAHA.109.572883

Grady, A., M. Carey and R. Sanson-Fisher, 2014. Assessing awareness of appropriate responses to symptoms of stroke. Elsevier J. Patient Educ. Counsel., 95: 400-405. DOI: 10.1016/j.pec.2014.03.007

Holtmann, C., M. Müller-Gorchs, A. Rashid, K. Weidenhaupt and C. Weinhardt et al., 2007. Medical opportunities by mobile IT usage-a case study in the stroke chain of survival. Proceedings of the 2nd European Conference on eHealth 2007, OFFIS Oldenburg, (HOO' 07), BibSonomy, pp: 115-129.

Kasner, S.E., 2006. Clinical interpretation and use of stroke scales. Lancet Neurology, Elsevier, 5: 603612. DOI: 10.1016/S1474-4422(06)70495-1

Khatri, P., D.O. Kleindorfer, S.D. Yeatts, J.L. Saver and J.P. Broderick et al., 2010. Strokes with minor symptoms: An exploratory analysis of the national institute of neurological disorders and stroke recombinant tissue plasminogen activator trials. Stroke, 41: 2581-2586. DOI: $10.1161 /$ STROKEAHA.110.593632
Nam, H.S., J.N. Heo, J.W. Kim, Y.D. Kim and J.H. Heo et al., 2014. Development of smartphone application that aids stroke screening and idetifying nearby acute stroke care hospitals. Yonsei Med. J., 55: 25-29. DOI: 10.3349/ymj.2014.55.1.25.

Nazifah, S.N., I.K. Azmi, B.B. Hamidon, I. Looi and M.R. Hanip, 2012. National Stroke Registry (NSR): Terengganu and Seberang Jaya Experience. Med. J. Malaysia, 67: 302-304.

NIHSS, 2013. National Institute of Health Stroke Scale.

NASAM, 2013. Stroke in Malaysia. National Stroke Association Malaysia.

Rajeev, M., 2013. Brain Attack. (n.d.) Mobile app to detect brain stroke symptoms. The Hindu.

Schwamm, L.H., R.G. Holloway, P. Amarenco, H.J. Audebert and L.R. Wechsler et al., 2009. A review of the evidence for the use of telemedicine within stroke systems of care: A scientific statement from the American heart association/American stroke association. Stroke, 40: 2616-2634. DOI: 10.1161/STROKEAHA.109.192360

SMSF, 2009-2013. Global market share held by smartphone operating systems from 2009 to 2013.

Stroke Centre, 2013. Stroke Association in United Kingdom.

Think, F.A.S.T., 2013. What is a stroke.

Urra, X., H. Ariño, L. Llull, S. Amaro and Á. Chamorro et al., 2013. The outcome of patients with mild stroke improves after treatment with systemic thrombolysis. PLoS One, 8: e59420-e59420. DOI: 10.1371/journal.pone.0059420

WebMD, 2012. The warning signs of stroke. WebMD Medical Reference.

Zandieh, A., Z.Z. Kahaki, H. Sadeghian, M. Fakhri and M. Ghabaee et al., 2013. A simple risk score for early ischemic stroke mortality derived from national institutes of health stroke scale: A discriminant analysis. Clin. Neurol. Neurosurgery, 115: 1036-1039. DOI: 10.1016/j.clineuro.2012.10.034 